\title{
Die Diskussion um die Euthanasie in Frankreich und der Schweiz vor dem Zweiten Weltkrieg
}

Von Hansjürg Zumstein

\section{Einleitung}

Im Frühsommer des Jahres 1987 forderte Jean-Marie le Pen, Präsident der französischen rechtsextremen «Front national», in der gutbeachteten Fernsehsendung «L'heure de verité», Aids-Kranke müßten eingesperrt werden ${ }^{1}$. Der Isolierungsvorschlag hat in Frankreich Tradition. Vor dem Zweiten Weltkrieg verlangten Le Pens Vorgänger ähnliche Maßnahmen gegen Tuberkulöse, deren Krankheit damals ebenfalls ansteckend und unheilbar war $^{2}$. Diese Forderung war Bestandteil der rechtsextremistischen Strömungen, die im damaligen Frankreich eine auffällige Resonanz fanden; auch die heutige «Front national» kann sich übrigens auf einen fast zehnprozentigen Wähleranteil stützen ${ }^{3}$.

Trotz solcher diskriminierenden Vorschlägen, eingebettet in ein Klima eines breit akzeptierten Rechtsradikalismus, wurde in Frankreich die planmäßige Vernichtung von Menschen nicht Praxis. Die Judenverfolgung blieb auf eine Mittäterschaft einer (zeitweise verhältnismäßig starken) Gruppe von Kollaborateuren beschränkt"; die Euthanasie von unheilbar Kranken erklärt sich teilweise durch kriegsbedingte Nahrungsmittel-Engpässe ${ }^{5}$.

In diesem Aufsatz sollen Erklärungshinweise für diese Entwicklung geliefert werden. Dazu werden wir vorerst die Komponenten der diskriminierenden Lehren des 19. und 20. Jahrhunderts bestimmen. Wir stützen uns dafür auf die Definitionen, die uns die Vertreter selbst liefern. Anschließend werden wir insbesondere die Laufbahn einer dieser Komponenten, der Euthanasie, weiterverfolgen. Wenn wir nämlich Klarheit gewinnen, wie Euthanasie, damals weitgehend als «Ausmerze» verstanden, diskutiert worden ist, werden wir Hinweise erhalten, die die nationale Laufbahn der diskriminierenden Lehren miterklären.

Beginnen wir mit den diskriminierenden Lehren des frühen 19. Jahrhunderts. Sie waren gekennzeichnet durch ein rassistisches Programm, das mehr oder weniger antisemitisch ausgerichtet war. Der herausragende Vertreter war der Franzose Joseph Arthur Comte de Gobineau, der mit seinem «Versuch über die Ungleichheit der Menschenrassen» (1854) seine Nachfol- 
ger maßgeblich beeinflußte. Der Rassismus bildet demnach die erste Komponente solcher Lehren.

Mit dem Aufkommen des Sozialdarwinismus erhalten ihre Vertreter in der zweiten Hälfte des 19. Jahrhunderts neues theoretisches Rüstzeug. Die Vielfalt an Strömungen, die sich in Inhalt und nationalem Erfolg unterscheiden, nimmt zu. Gemeinsam ist ihnen, dass sie der Sozialdarwinismus, parallel zu seinen Schlüsselbegriffen «positive und negative Auslese», mit zwei weiteren Komponenten bestückt: der Eugenik und der Euthanasie. Die Eugenik steht dabei für die «positive Auslese» oder die sogenannte «Selektion durch natürliche Zuchtwahl». Demgegenüber steht die Euthanasie als «negative Auslese», d.h. als Selektion durch gezielte Tötung von unerwünschten Menschen.

Diese Euthanasiediskussion soll uns nun im Fall Frankreich miterklären, weshalb seine rechtsextremen Strömungen kaum eine grausame Praxiswirkung hatte. Vorgängig werden wir an einem Fallbeispiel aus dem Schweizer Kanton Bern untersuchen, ob dieses Thema in diesem Zeitraum auf ein nichtartikuliertes Einverständnis stieß. Diese beiden Ansätze haben wir eingehender in unserer Arbeit «Le débat sur l'Euthanasie avant la deuxième Guerre mondiale» ${ }^{6}$ sowie in einem Aufsatz über die Euthanasie-Diskussion im Schweizer Kanton Bern untersucht ${ }^{7}$.

\section{Definition und Wandel des Begriffes Euthanasie}

Der Inhalt des Begriffes «Euthanasie» geht bereits aus dem vorhergehenden Kapitel hervor. Wir möchten ihn nachfolgend noch präzisieren. Ursprünglich steht der Begriff für den «schönen, leichten Tod». Im 19. Jahrhundert erweitert sich die Bedeutung von Euthanasie. Der Begriff umfaßt nun alle Methoden, die den Todeskampf abkürzen und von einer schmerzvollen, unheilbaren Krankheit erlösen sollen ${ }^{8}$. Während der Jahrhundertwende erhält das Wort zusätzlich die Bedeutung einerseits von «Tötung auf Verlangen» (homicide sur demande), andererseits von «Tötung mit Einwilligung» (suppression des «existences inutiles»).

Die beiden neuen Begriffsinhalte sind praktisch kaum trennbar. In unserer Arbeit über die französische Euthanasie-Diskussion untersuchten wir deshalb beide Bereiche. In diesem Aufsatz konzentrieren wir uns allerdings auf die Euthanasie-Diskussion im Sinne der «Vernichtung lebensunwerten Lebens», also der «Tötung ohne Einwilligung». 


\section{Ein Fallbeispiel: Parlaments-Diskussion im Kanton Bern}

Wie bereits erwähnt, untersuchten wir eine Debatte im Berner Kantonsparlament, in der die «Tötung der unheilbaren Geisteskranken und Idioten» diskutiert wurde. Der auslösende Vorstoß war im Jahr 1922 eingereicht worden, die eigentliche Debatte fand im drauffolgenden Jahr statt; also zwei Jahre nachdem die Schrift von Binding und Hoche erschienen ist, die den deutschen und ausländischen Diskurs maßgeblich beeinflußt hatte ${ }^{9}$. Auch der Verfasser des kantonalbernischen Vorstoßes, Kantonsarzt Alfred Hauswirth (1872-1959), schien davon geprägt, finden sich doch Bindingsche Kategorisierungen in seinen parlamentarischen Ausführungen wieder.

Der verbindliche Vorstoß (Motion) wurde erheblich erklärt, allerdings nur, weil er hauptsächlich eine Revision der bestehenden Gesundheitsgesetzgebung verlangt und den Vernichtungsvorschlag nicht explizit erwähnt hatte. Der Hauswirthsche Vorstoß löste im übrigen keine gesetzgeberischen Arbeiten aus.

Im Zusammenhang mit unserer Ausgangsthese ist interessant zu analysieren, wie der Vorstoß von den politischen Exponenten rezipiert worden ist. Dadurch sind eventuell Hinweise zu gewinnen für die Erklärung, weshalb die Vernichtungsdebatte nicht dieselbe Ausbreitung fand wie in Deutschland.

Vorerst ist zu bemerken, daß der parlamentarischen Diskussion über Euthanasie Ausnahmecharakter zukommt. Wir sind im Rahmen unserer Arbeiten auf drei weitere Parlamentsdebatten gestoßen ${ }^{10}$.

Was die Person des Motionärs betrifft, so handelt es sich um eine Schlüsselfigur des politischen Leben Berns. Hauswirth war Gründer der stadtberner Sektion der Bauern-, Gewerbe- und Bürgerpartei (BGB) ${ }^{11}$ und prägte dementsprechend die Politik. Er arbeitete als Stadt- und zeitweise als Kantonsarzt, d.h. er fungierte als Chefbeamter im öffentlichen Gesundheitswesen. Dies zu einer Zeit, in der zahlreiche Bürger unter gesundheitlich kritischen Bedingungen leben mußten (überfüllte und unhygienische Wohnung, Slumcharakter vieler Altstädte, offensichtlich gesundheitschädliche Arbeitsbedingungen). Sein Arbeitsberich besaß deswegen eine allgemein anerkannte Bedeutung.

Hauswirth begründete seinen Vorstoß, Geisteskranke und Idioten zu töten, erstens mit Erbarmen: «Mit dem unheilbar kranken Tier hat man Erbarmen, aber mit dem unheilbaren Geisteskranken (...) hat man kein Erbarmen.» ${ }^{12}$ Zweitens argumentierte er mit der öffentlichen Belastung, die 
diese Menschen darstellen würden, und drittens sprach er von der Gefahr, die von ihnen ausginge: «Frei umherlaufende unzurechnungsfähige Irre und Idioten begehen abscheuliche Verbrechen.» ${ }^{13}$

Die Reaktionen in Rat und Medien reichten von Entrüstung bis zu Verständnis. Am deutlichsten und argumentativ eigenwilligsten protestierte die protestantische Kirche (Staatskirche des Kantons Bern). Die sozialdemokratische Tageszeitung «Tagwacht» äußerte dagegen Verständnis, forderte allerdings, vorerst müßten Präventivmaßnahmen gestützt auf eugenische Erkenntnisse getroffen werden. Das konservative «Berner Tagblatt» begrüßte den Hauswirthschen Vorschlag und regte an, ihn auf Verbrecher auszudehnen. Der liberale «Bund» pendelte zwischen ablehnender und zustimmender Position (je ein Kommentar). Ähnlich unbestimmt verhielt sich auch die andere damals bedeutende Berner Zeitung, die konservative «Neue Berner Zeitung». Die Parlamentarier verwarfen den Vorschlag, ein gewisses Verständnis äußerte einzig ein sozialdemokratischer Abgeordneter. Parlamentsintern scheint Hauswirth allerdings auf Zustimmung gestoßen zu sein. Er schreibt: «Schade, daß diejenigen Ratsmitglieder, die mir nachher uneingeschränkte Übereinstimmung mit meinen Ansichten ausgesprochen, dies nicht vorher, im Rate selbst taten, es hätte dies ein ganz anderes Bild gegeben!» ${ }^{14}$

Einen «Sturm der Entrüstung» jedenfalls, wie die öffentliche Reaktion nach Kriegsende dargestellt wurde ${ }^{15}$, löste der Vorschlag nicht aus. Es kann daraus geschlossen werden, daß in gewissen Kreisen eine nichtartikulierte Akzeptanz für solche Vernichtungsideen bestanden hatte. Indiz dafür ist auch die breite Zustimmung, die die damalige Diskriminierung der Zigeuner fand, betrieben durch die offiziöse Organisation «Pro Juventute». ${ }^{16}$

Die Diskussion des Hauswirthschen Vorschlags ist ein Fallbeispiel. Wir haben die weitere nationale Euthanasie-Diskussion nicht untersucht. Schlußfolgerungen sind nur beschränkt möglich. Gleichzeitig ist zu betonen, daß diese Diskussion ein isoliertes Phänomen blieb. Sie fand kaum Echo in den Nachbarkantonen ${ }^{17}$.

\section{Die Diskussion in Frankreich}

Zuverlässigere Aussagen können wir hingegen über die Diskussion in Frankreich machen. Wir untersuchten die dortige Euthanasie-Debatte vom Jahrhundertbeginn bis zum Jahr 1939. Wir unterschieden zwei Perioden, 
zunächst eine erste bis zum Ende des Ersten Weltkriegs und anschließend eine zweite, die unmittelbar daran anschließt.

Während der ersten Periode strukturierte sich das Thema Euthanasie, hauptsächlich als «Vernichtung lebensunwerten Lebens» verstanden. In der zweiten Periode hingegen verschwindet dieser Inhalt fast völlig, es wird einzig diskutiert über Euthanasie im Sinne des «Tods auf Verlangen». Die Gründe dieses Verschwindens vermuten wir in einer für Frankreich charakteristischen Reserviertheit in juristischen, religiösen und medizinischen Kreisen diesem Thema gegenüber.

Diese Haltung läßt sich an der Person des Arztes François Guermonprez illustrieren. Guermonprez (1849-1932) war erster Präsident der Action française von Nordfrankreich, mithin also ein Vertreter jener rassistischen, antisemitischen Lehre. Zudem war der überzeugte Royalist in ideologische Streitigkeiten innerhalb der französischen katholischen Kirche verwickelt; er bekämpfte energisch den Sozialkatholizismus. Doch wie andere Mitglieder der rechtsextremen Action française war Guermonprez ein erbitterter Gegner der Euthanasie. Allein der Titel seines bekanntesten Werkes, das sich mit diesem Thema auseinandersetzt, ist aufschlußreich: «L'assassinat médical» heißt das 1904 publizierte Buch. Den Titel verwendete der Medizinprofessor (er lehrte an der katholischen Musteruniversität Faculté catholique de médicine de Lille) noch für einen Zeitungsartikel, abgedruckt im «Journal des Sciences médicales de Lille». ${ }^{18}$ Sein Engagement gegen die Euthanasie erklärt sich aus seinem katholischen Menschenbild, aus dem heraus er folgende medizinische Ethik definierte: «Der Arzt muß, solange er kann, helfen; trotzdem erleichtern; immer trösten; nie töten.» Dieses Leitprogramm wird von nachfolgenden Euthanasie-Gegnern stets aufgegriffen werden, meist ohne Quellenangabe und dem medizinischen Fortschritt entsprechend mit stärkerer Betonung der Hilfe (Therapie).

Mit der Biographie Guermonprez' wird der Sinn der Drei-KomponentenTheorie deutlich. Sie hilft verstehen, weshalb rassistische Strömungen hinsichtlich Eugenik und Euthanasie in Frankreich nicht dieselbe Wirkung entfalten wie in Deutschland.

Eine vertiefte Analyse der Euthanasie-Diskussion in dieser ersten Periode zeigt weiter, daß wir in Frankreich - anders als in Deutschland - keine juristische, wohl aber eine medizinische Diskussion vorfinden. Allerdings ist das Wort «Diskussion» teilweise übertrieben, denn es finden sich wenig Texte, die sich zu Euthanasie im oben definierten Sinn äußern. Wir finden eine geringe Anzahl Artikel in medizinischen oder pseudomedizinischen 
Zeitschriften mit oft starker Verbreitung, die unwidersprochen Euthanasie mit der Begründung «soziale Verteidigung» auf Individuen anzuwenden verlangen, die der Gesellschaft zur Last fallen würden.

Wir können zusammenfassen: Obwohl die Verbreitung dieser Zeitschriften beachtlich ist, spricht die Tatsache, daß die fraglichen Artikel unwidersprochen blieben, gegen eine intensive Diskussion. Die andere mögliche Folgerung, eine allgemeine Übereinstimmung mit den Artikelinhalten, können wir auschließen, wie der weitere Verlauf der Euthanasie-Diskussion zeigen wird. Wir stellen aber fest, daß in dieser ersten Periode bisherige ethische Konzeptionen stark erschüttert worden sind. Das zeigt sich beispielsweise darin, daß fast alle Autoren, ob für oder gegen Euthanasie, historisch weit zurückliegende Ethiken als vorbildlich beschrieben. Oft ist dies Sparta, wo anscheinend «Vernichtung von lebensunwertem Leben» Usanz war. Zudem bemerkten wir, teilweise zusammenhängend mit dieser ethischen Lehre, daß die Vorstellungen über die «Vernichtung lebensunwerten Lebens» inkoherent waren (unterschiedliche Kategorisierungen). Gleichzeitig führten aber die Autoren bereits eine Auswahl von diskriminierendem Vokabular ein, das in Deutschland in dieser Breite erst nach der Schrift von Binding und Hoche entstand (1920).

Doch trotz der Präsenz dieses Vokabulars verschwand die EuthanasieThematik im Sinne der «Vernichtung lebensunwerten Lebens» in der zweiten Periode fast völlig. Statt dessen befaßte sich die Öffentlichkeit leidenschaftlich mit dem Thema des «Tods auf Verlangen». Höhepunkte dieser Diskussion war eine Pariser Aufführung des Stückes «L'Euthanasie ou le devoir de tuer» (1923) von René Berton ${ }^{19}$ sowie die ersten Praxisfälle (1924), die ein lebhaftes Echo in der Tagespresse auslösten. Diese Diskussion und ihre Begleitumstände sind ausführlich behandelt in unserer Arbeit; hier möchten wir uns aber auf die Diskussion des anderen Euthanasie-Inhalts konzentrieren.

Wenn wir das Verschwinden dieser Diskussion verstehen wollen, müssen wir die sozio-ökonomische Situation der Ärzteschaft, in dieser Periode in Deutschland gewichtige Wortführerin der Vernichtungsideen, untersuchen. Wir stellen dabei fest, daß der französische Arzt im Gegensatz zu seinem deutschen Kollegen nahezu keine wirtschaftlichen Sorgen kannte. Er blieb von der Krise der dreißiger Jahre praktisch unberührt ${ }^{20}$. Zusätzlich vertiefte sich in dieser Epoche seine schon bei Guermonprez beobachtete Bindung zum Katholizismus. Dies war eine Folge der katholischen «conquêtes» mit der Bewegung «Action catholique».21 
Zu diesen Unterschieden kommen die grundsätzlichen Differenzen zwischen Frankreich und Deutschland. Wir stellen zuerst eine abweichende demographische Situation fest, die wiederum die offizielle Familienpolitik in eine Richtung drängte, die gegensätzlich zu jener Deutschlands war. Frankreich war nämlich während den Jahren 1919 bis 1939 gekennzeichnet durch ein damals neues Phänomen: die demographische Krise, ausgelöst durch einen empfindlichen Geburtenrückgang ${ }^{22}$. Diese favorisierte eine Familienpolitik, die sich wesentlich am Beispiel des Sozialkatholiken Léon Harmel orientierte (dieser Unternehmer führte erstmals Kinderzulagen ein). Ab 1930 wird diese Politik offizialisiert ${ }^{23}$, während gleichzeitig in Deutschland die Familienpolitik allmählich rassistisch ausgerichtet wird.

Zweiter wichtiger allgemeiner Unterschied ist die Wirtschaftskrise, die Frankreich wesentlich weniger hart traf als Deutschland, wo sie noch verstärkt wurde durch politische Krisen ${ }^{24}$. In Frankreich hingegen stieg die Kaufkraft allgemein an, selbst für die Arbeitslosen blieb sie stabil ${ }^{25}$.

Der dritte Unterschied ist kultureller Natur. Norbert Elias diagnosziert, daß die Deutschen sich allgemein vor der Uneinigkeit fürchteten; ein Resultat der jahrhundertelangen innerdeutschen Streitigkeiten. Diese «traumatische Furcht vor der traditionellen Uneinigkeit» erleichterte laut Elias die Etablierung der Nazi-Herrschaft; sie erklärt, weshalb eine Ideologie stärker rezipiert wird, die das gesellschaftliche Dasein scheinbar vereinfachte, indem sie das Prinzip des Utilitarismus zum Maßstab für alles machte ${ }^{26}$. In Frankreich hingegen war man gewohnt, mit verschiedenen sozialen Widersprüchen zu koexistieren, deshalb fand sich keine Mehrheit für utilitaristische Ideen.

Diese drei Unterschiede kombiniert mit der spezifischen Situation der französischen Ärzte sind Erklärungsansätze für das Verschwinden dieser Euthanasie-Diskussion. Daraus folgt, daß dem Rechtsextremismus die Komponente Euthanasie fehlte (über die Eugenik-Thematik können wir nichts aussagen, da wir sie nicht untersucht haben). Diese fehlende Thematisierung erklärt teilweise, weshalb die Rechtsextremisten Frankreichs in den vierziger Jahren, unter dem Schutz der Nazis, sich auf antisemitische Aktionen konzentrierten und keine Eugenik- und Euthanasie-Maßnahmen unternahmen ${ }^{27}$. 


\section{Anmerkungen}

1 Le Monde, 8. Mai 1987

2 vgl. z. B. Regnault Jules, «Assassinat médical ou suprême charité?», in: La Revue, 15 juin 1905. Er fordert Landkolonien für Tuberkulöse und begründete: «Quant aux incurables, leur mort serait peut-être avancée, mais au point de vue social, leur disparation serait un bien.»

3 Hauptpfeiler des rechtsextremistischen Denken in Frankreich vor dem Zweiten Weltkrieg war die «Action française», die sich hauptsächlich durch Antisemitismus und Ablehnung der Demokratie determinierte. Bis zu ihrer Verurteilung durch den Papst Pius XI. im Jahr 1926 prägte sie wesentlich die Haltung der französischen Katholiken.

4. Mindestens $85 \%$ der 75721 aus Frankreich deportierten Juden sind von der französischen Polizei festgenommen worden. Vgl. Le Monde, 11. Mai 1985, «Convois pour la mort» von Serge Klarsfeld.

5 Gemäß neusten Forschungen hat in Frankreich eine solche Euthanasie in den psychiatrischen Anstalten stattgefunden. Rund 40000 Kranke sollen während den Kriegsjahren in den Psychiatriespitälern verhungert sein. Diese Hochrechnung macht Max Lafont in seiner Untersuchung über den Kriegsalltag in der Psychiatrieanstalt Vinatier (bei Lyon). Lafont Max, L'Extermination douce. Thèse. Ed. AREFPPI, Domaine de Clermont, le Cellier, 44850 Ligné. 225 p.

6 Zumstein Hansjürg, Le débat sur l'Euthanasie avant la deuxième Guerre mondiale. Mémoire préparé sous la direction de Monsieur le Professeur Mayeur, Université Paris IV, 1986. $109 \mathrm{p}$.

7 Zumstein Hansjürg, Die Diskussion über die Euthanasie in der deutschen Schweiz in den zwanziger Jahren. Seminararbeit Geschichte, eingereicht bei Frau Professor Beatrix Mesmer, Universität Bern, 1985. 59 S.

8 Grand Dictionnaire encyclopédique Larousse. Paris 1983, tome 6, p. 4029.

9 Binding Karl/Hoche Alfred, Die Freigabe der Vernichtung lebensunwerten Lebens. Ihr Maß und ihre Form. Verlag Felix Meiner, Leipzig 1920.62 S.

101903 im sächsischen Parlament, 1913 im Deutschen Reichstag, 1936 im englischen House of the Lords; vgl. Charles Raymond, Peut-on admettre l'euthanasie? Paris 1955. 194 p., p. 16-17.

11 Konservative Partei, die unter dem Namen Schweizerische Volkspartei (SVP) noch heute entscheidend die kantonalbernische Politik bestimmt.

12 Tagblatt (Parlamentsprotokoll) des Kantons Bern, 1923, S. 190.

13 Tageszeitung «Bund», 23. September 1923, S. 6.

14 «Bund», 23. September 1923.

15 Tagblatt des Kantons Bern, 11. Mai 1949, S. 254: Politiker Ernst Steinmann über das neue Gesundheitsgesetz.

16 Die Pro-Juventute-Internierungspolitik von Zigeunerkindern stützte sich auf die eugenische Lehre. Die Folgen dieser «Pro-Juventute»-Aktionen, die bis in die siebziger Jahre andauerten, passionieren noch heute die Schweizer Öffentlichkeit. Erst anfangs Sommer 1987 einigte sich die Organisation «Pro Juventute» mit Zigeunervertretern, wie diese für das begangene Unrecht materiell zu entschädigen und wie die Akten zu behandeln seien, vgl. «Berner Zeitung», 8. Mai 1987. 
17 Eine Untersuchung der beiden überregionalen Blätter «Neue Zürcher Zeitung» und «Vaterland» (katholisch-konservativ) blieb ohne Resultat.

18 15. März 1904, S. 233-243 und S. 329-342.

19 Berton René, L'Euthanasie ou le devoir de tuer. Drame en un acte. Ed. Librairie théâtrale, Paris 1925. 34 p.

20 Im Gegenteil: «Le Prix de la consultation ne baisse pas, il monte même parfois.» Vgl. Raoux François, Naissance de la Corporation médicale, 1789 à 1943. Thèse médicale. Paris 1979. 143 p., p. 100.

21 Frankreich war in dieser Zeit geprägt vom Entstehen der «Action catholique», es war die Epoche der «conquêtes». Etwas vereinfacht gesagt bedeutete dies, daß Angehörige jeder sozialen Schicht und Generation sich zu einer katholischen Aktionsgruppe formierten, die teilweise äußerst aktiv Neumitglieder worben. Auch die Ärzte besassen ihre «Action catholique», die sich übrigens explizit distanzierte von der deutschen Nazi-Medizin (im Jahr 1936, also noch vor Bekanntwerden der Sterilisations-Praxis).

22 Trotignon Yves, La France au $\mathrm{XX}^{\mathrm{e}}$ siècle. Chapitre «La crise démographique». Ed. Dunod, Paris 1985, 444 p., p. $120 \mathrm{ff}$.

23 Trotignon, La France au XX $\mathrm{XX}^{\mathrm{e}}$ siècle, p. 128/9.

24 z. B. Ende 1929/Anfang 1930: Stresemann stirbt im Oktober, im Dezember demissioniert Finanzminister Hilferding, im März 1930 demissioniert Reichsbankdirektor Schacht, kurz darauf stürzt die Regierung Müller über die Arbeitslosenkassen-Frage.

25 Prost Antoine in seiner Vorlesung «Le Mouvement ouvrier en France au XX $\mathrm{X}^{\mathrm{e}}$ siècle», Sorbonne 1985/86. Einführung: Front populaire, 17.12.1985.

26 Elias Norbert, Die höfische Gesellschaft. Suhrkamp, 1983. 456 S., S. 410-414.

27 Es sei nochmals darauf hingewiesen, daß der Nazi-Euthanasie und -Eugenik in den zwanziger und dreißiger Jahren breite Diskussionen vorausgegangen sind.

\section{Summary}

The author has examined the debate on euthanasia before World War II in France and Switzerland. At that time, euthanasia was largely understood as the «extermination of life not worth living». He states that the two debates differed in content from that in Germany which ultimately led to the National Socialist programme for the extermination of the sick and infirm. With regard to contents, the main difference was that although in France there was indeed a strong racist and antisemitic trend, this was not supplemented by a broad debate on the «extermination of live not worth living». The peripheral significance of the theme of euthanasia - in the sense of extermination - which is also detectable for Switzerland, provides indications as to how the different attitudes during the war might be explained.

Hansjürg Zumstein

Stauffacherstraße 1

CH-3014 Bern 